\title{
Effect of ABCE1-silencing gene, transfected by electrotransfer, on the proliferation, invasion, and migration of human thyroid carcinoma SW579 cells
}

\author{
X. Qu ${ }^{1,2}$ and L. Zhang ${ }^{1}$ \\ ${ }^{1}$ ENT and Maxillofacial Oncology, Tianjin Medical University Cancer Hospital, \\ Tianjin, China \\ ${ }^{2}$ Thyroid Breast Surgery, \\ Fourth Clinical College of Tianjin Medical University Center, Tianjin, China \\ Corresponding author: L. Zhang \\ Email: zhanglunphd@126.com
}

Genet. Mol. Res. 14 (4): 14680-14689 (2015)

Received March 4, 2015

Accepted July 22, 2015

Published November 18, 2015

DOI http://dx.doi.org/10.4238/2015.November.18.32

ABSTRACT. We investigated the effect of an ABCE1-silencing gene on the proliferation, invasion, and migration of human thyroid carcinoma SW579 cells. We designed and synthesized targeted $A B C E 1$-siRNA sequences and a negative control sequence (NC-siRNA), and transfected them into human thyroid cancer SW579 cells by electrotransfer to obtain ABCE1SW579 and NC-siRNA-SW579 cells (siRNA is small interfering RNA). Through reverse transcription polymerase chain reaction and western blotting analysis, $A B C E 1 \mathrm{mRNA}$ and protein expression levels in the electrotransferred cells were detected, and flow cytometry was used to detect cell cycle and apoptosis. The cell counting kit-8 (CCK-8) proliferation assay, the scratch healing assay, and the cell invasion assay were used to measure cell proliferation, migration, and invasion capabilities, respectively. Compared with NC-siRNA-SW579 and Ctrl-SW579 groups, ABCE1 mRNA and protein expression levels in the ABCE1-SW579 cells were significantly 
reduced. The growth rate of ABCE1-SW579 cells was significantly inhibited, the cell cycle was arrested in the G0/G1 phase, and the number of cells in the S phase was reduced. Compared with the Ctrl-SW579 group, the cell apoptosis rate in the ABCE1-SW579 group was significantly higher $(P<0.01)$, and proliferation, migration, and invasion were significantly reduced $(P<0.05)$. Expression of the ABCE1-silencing gene, transfected by electrotransfer, could inhibit the proliferation, invasion, and migration of thyroid cancer cells.

Key words: Cell proliferation; Thyroid cancer; ABCE1; Electrotransfer; SW579 cells

\section{INTRODUCTION}

Thyroid cancer is a common malignancy of the head and neck, accounting for $1-2 \%$ of systemic cancers. It can occur at any age, although onset is most frequent in the 30-40 year age bracket, and is more common in women. It has a long incubation period and a slow growth rate, but it often features a single metastasis at an early stage. Studies have shown that the proliferation, invasion, and migration of the tumor are closely related to gene regulation, which could provide a novel and effective means of tumor treatment (Chen et al., 2012). Studies have also shown (Pisareva et al., 2011) that the ATP-binding cassette E1 (ABCE1) protein, which is a member of the subfamily of ATP-binding cassette transporters, can inhibit cell apoptosis by interfering with intracellular ribonuclease $\mathrm{L}$ (RNase $\mathrm{L}$ ) activity, and promote cell proliferation and differentiation. It is closely related to the biological behavior of tumors, such as tumor proliferation, invasion, and migration. Thus, theoretically, blocking the expression of ABCE1 in tumor cells should be an effective tumor treatment. There are few reports on the relevance of $A B C E 1$ genes to proliferation, invasion, and migration of thyroid cancer worldwide. In this study, we designed and synthesized ABCE1-siRNA sequences (siRNA is small interfering RNA) and transfected them into human thyroid cancer SW579 cells by electrotransfer to obtain an ABCE1-silencing gene, and explored its impact on the biological behavior of thyroid SW579 cells.

\section{MATERIAL AND METHODS}

\section{Cell lines and reagents}

Thyroid cancer SW579 cells were purchased from the China Type Culture Collection Center (Collection Center of Wuhan University), Dulbecco's modified Eagle's medium (DMEM), Opti-MEM, TRIzol, and trypsin were purchased from Invitrogen, USA; fetal bovine serum (FBS) was purchased from Gibco, USA; the plasmid pcDNA3.1 was purchased from the Invitrogen Corporation; an L plasmid extraction kit, RNA extraction reagent TRIzol, and a DNA gel purification and recovery kit were purchased from the Beijing Tiangen Biotech Company; DNA marker and reverse transcription polymerase chain reaction (RT-PCR) reagents were purchased from the TaKaRa Company, USA; a cDNA synthesis kit was purchased from the Japan TOYOBO Company; an AMV reverse transcriptase kit was purchased from Hangzhou Bioer (Hangzhou, Zhejiang, China); the RNA interference sequences were synthesized by Zhuhai Yingping Biological Technology 
Co., Ltd.; the PCR primers were synthesized by Shanghai GenePharma Biotech Co., Ltd.; a cell counting kit-8 (CCK-8) was purchased from the China Beyotime Biotech Co.; rabbit anti-human ABCE1 polyclonal antibody and mouse anti-human glyceraldehyde 3-phosphate dehydrogenase (GAPDH) monoclonal antibody were purchased from Santa Cruz, USA; horseradish peroxidaselabeled goat anti-rabbit IgG was purchased from the Beijing Zhongshan Company; an enhanced chemiluminescence kit was purchased from the Thermo Company, CA, USA; Matrigel gel was purchased from BD Biosciences; and polyvinylidene difluoride membranes were purchased from the Bio-Rad Company, USA.

\section{Design and synthesis of $A B C E 1$-targeted siRNA}

The $A B C E 1$-targeted $A B C E 1$-siRNA sequences were designed according to the literature (Tian et al., 2012). The sense sequence was: 5'-ATCCGCTACAGCGAGTACGTTTACCTGTGAAGCCACAGATGGGGTAAACGTACTCGCTTAGCTTTTTTG-3' and the antisense sequence was: 5'-AATFCAAAAAAGCTACAGCGAGTACGTTTACCCCATCTGTGGCTTCACAGGTAAAC GTACTCGCTGTAGCG-3'; the sense sequence of the non-homologous negative control siRNA (NC-siRNA) was: 5'-GATCCGCGAGACCTCAGTATGTTACCTGTGAAGCCACAGATGGGGT AACATACTGAGGTCTCGCTTTTTTG-3', and the antisense sequence was: 5'-AATTCAAAAAAG CGAGACCTCAGTATGTTACCCATCTGTGGCTTCACAGGTAACATACTGAGGTCTCGCG-3'. All sequences were synthesized by Zhuhai Yingping Biotechnology Co., Ltd.

\section{SW579 cells transfected with ABCE1-siRNA by electrotransfer}

Thyroid cancer SW579 cells were cultured in DMEM containing $10 \% \mathrm{FBS}\left(37^{\circ} \mathrm{C}, 5 \% \mathrm{CO}_{2}\right)$. Cells in the logarithmic growth phase were made into a single cell suspension and washed with phosphate-buffered saline (PBS). After trypsin digestion and centrifugation, the electrotransfer solution was added and the cells were resuspended. The cells $(800 \mathrm{~g})$ were centrifuged for $5 \mathrm{~min}$, washed three times, centrifuged again, and resuspended in power-transfer fluid. The cells were then mixed with $10 \mu \mathrm{g} A B C E 1$-siRNA plasmid in an electrical cup and placed on ice for $0.5 \mathrm{~h}$ before electroporation (voltage $450 \mathrm{~V} / \mathrm{cm}$, capacitance $25 \mu \mathrm{F}$, time $0.9 \mathrm{~ms}$ ). The transfected cells were named ABCE1-SW579 cells, and were stored at room temperature for $0.5 \mathrm{~h}$. The cells were then transferred to DMEM (containing $10 \%$ calf serum and $1 \%$ double-antibody) on a Petri dish and incubated in an incubator box $\left(37^{\circ} \mathrm{C}, 5 \% \mathrm{CO}_{2}\right)$. Cells subjected to electrotransfer with an empty vector were taken as the control (named as Ctrl-SW579 cells). RNA interference effects were detected after $48 \mathrm{~h}$.

\section{Expression of ABCE1 mRNA detected by RT-PCR}

Cells from the ABCE1-SW579 group, the NC-siRNA-SW579 group, and the Ctrl-SW579 group ( $1 \times 10^{7}$ from each group) were collected and washed with PBS three times. Total RNA was extracted using TRIzol and cDNA was synthesized by reverse transcription PCR amplification. The upstream primer of ABCE1 was 5'-TTGGTTGTGGGAAGTCGT-3', and the downstream primer was 5'-GCTTATGTAGTTAATGGGAGGT-3'; the amplified product had $415 \mathrm{bp}$. The upstream primer of GAPDH was: 5'-GAGTCAACGGATTGGTCGT-3', and the downstream primer was: 5'-GACAAGCTTCCCGTTCTCAG-3'; the amplified product had $189 \mathrm{bp}$. The RT-PCR regimen was: 
pre-degeneration at $95^{\circ} \mathrm{C}$ for $5 \mathrm{~min}$; degeneration at $95^{\circ} \mathrm{C}$ for $30 \mathrm{~s}$; annealing at $60^{\circ} \mathrm{C}$ for $30 \mathrm{~s}$; and extension at $72^{\circ} \mathrm{C}$ for $1 \mathrm{~min}$, for a total of 35 cycles. The PCR products were analyzed by $1.2 \%$ agarose gel electrophoresis and observed using a UVI gel imaging system. The Image-Pro plus 7.0 software was used to analyze the stripe gray values, and relative expression levels of $A B C E 1$ mRNA were measured using the ratio $A B C E 1 / G A P D H$.

\section{Expression of ABCE1 protein detected by western blot}

Cells $\left(1 \times 10^{7}\right)$ from each group were collected and washed with PBS three times, and total protein was isolated and detected. Protein $(60 \mu \mathrm{g})$ was separated by $10 \%$ sodium dodecyl sulfate polyacrylamide gel electrophoresis, and electrotransferred to a polyvinylidene difluoride membrane. After soaking in $5 \%$ skimmed milk for $2 \mathrm{~h}$ at $37^{\circ} \mathrm{C}$, the protein was incubated with the first antibody-ABCE1 polyclonal antibody (1: 1000) for $1 \mathrm{~h}$. After washing in Tris-buffered saline (4X $15 \mathrm{~min}$ ), it was incubated at room temperature for $2 \mathrm{~h}$ at $37^{\circ} \mathrm{C}$ with the second antibody-horseradish peroxidase-labeled antibody (1:5000) and $\mathrm{GAPDH}$, and then washed with PBS before detection by enhanced chemiluminescence. X-rays tablet, developing, and fixing were conducted. A UVI gel imaging system was used for photography and the Image-Pro plus 7.0 software was used to analyze the stripe gray values, with the ratio ABCE1/GAPDH representing the relative expression of ABCE1 protein.

\section{Cell cycle detection of SW579 cells using flow cytometry}

Cells from each group were collected and the cell density was adjusted to $1 \times 10^{6}$ cells/L. They were then washed with pre-cooled PBS twice. The cell pellet was mixed with $70 \%$ cold ethanol at $4^{\circ} \mathrm{C}$, and the cells were washed and adjusted to a density of $1 \times 10^{6} / \mathrm{mL}$, then incubated with Tris- $\mathrm{HCl}$ buffer $(\mathrm{pH} 7.4)$ containing $50 \mu \mathrm{g} / \mathrm{mL}$ RNA enzyme for $30 \mathrm{~min}$. The cells' DNA was stained with $1 \mu \mathrm{g} / \mathrm{mL}$ propidium iodide and stored in the dark at room temperature for $30 \mathrm{~min}$. Flow cytometry was used to detect cell cycle and apoptosis. The experiment was repeated three times.

\section{Proliferation of SW579 cells detected by the CCK-8 assay}

SW579 cells were seeded on 96-well plates with a density of 4000 cells/well, and then 200 $\mu \mathrm{L}$ DMEM culture containing $10 \%$ FBS was added. There were six wells in each group and a blank well served as a control. The CCK-8 $(20 \mu \mathrm{L})$ was added to each well and after incubation for $4 \mathrm{~h}$, the absorbance at $490 \mathrm{~nm}$ [D (490)] of each well was detected using a microplate reader and the growth curve was drawn.

\section{Migration of SW579 detected by the scratch healing assay}

The three kinds of cells described above in their logarithmic growth phase were seeded on 6 -well plates at a density of $5 \times 10^{3}$ cells/well. When the cells reached a confluence of $90 \%$ in each group, a scratch was administered with a $10-\mu \mathrm{L}$ pipette dropper or a sterile toothpick along the cell monolayer. After washing with 0.01 M PBS three times, $2 \mathrm{~mL}$ Roswell Park Memorial Institute (RPMI) 1640 medium was added to each well for continuous incubation $\left(37^{\circ} \mathrm{C}, 5 \% \mathrm{CO}_{2}\right)$. After 48 $\mathrm{h}$, the cells were inverted under a microscope to observe their migration and to photograph them. 


\section{Transwell invasion assay}

A polycarbonate microporous membrane was capped with Matrigel at a density of $50 \mu \mathrm{g} /$ well; in the polymerized lower chamber, 10\% FBS was added as the culture medium and $100 \mu \mathrm{L}$ of the SW579 cell suspension described above (the total number of cells was $3 \times 10^{5} / \mathrm{L}$ ) from each group was added to the upper chamber, which was placed in an incubator for $24 \mathrm{~h}$. After fixing with $4 \%$ paraformaldehyde for $10 \mathrm{~min}$, cells were stained with hematoxylin for $20 \mathrm{~min}$, and then cells on the lower surface of the membrane were counted using a light microscope. Penetrating cells in five random observation of each membrane were counted and the average was calculated. Each group was arranged in three parallel chambers and the operation was repeated three times. Cell invasion rate $(\%)=$ total number of penetrating cells / total number of cells inoculated on upper chamber $\times 100 \%$.

\section{Statistical analysis}

Data were analyzed the using the SPSS 16.0 statistical software. The count data are reported as means $\pm \mathrm{SD}$. Differences among groups were compared using the Q-test and one-way analysis of variance. $P$ values of $<0.05$ or $<0.01$ were considered to be statistically significant.

\section{RESULTS}

\section{$A B C E 1$-siRNA inhibition of $A B C E 1$ mRNA expression in SW579 cells}

RT-PCR detection (Figure 1) showed that ABCE1 mRNA expression in the ABCE1SW579 group was significantly reduced compared with the NC-siRNA-SW579 and Ctrl-SW579 groups, and the differences were statistically significant $[(0.44 \pm 0.03)$ vs $(0.68 \pm 0.07),(0.70 \pm$ $0.09), P<0.05]$. The Ctrl-SW579 and NC-siRNA-SW579 groups showed no significant difference in $A B C E 1$ mRNA expression ( $P>0.05$ ), which indicates that $A B C E 1$-siRNA transfection effectively suppresses $A B C E 1$ mRNA expression in SW579 cells.
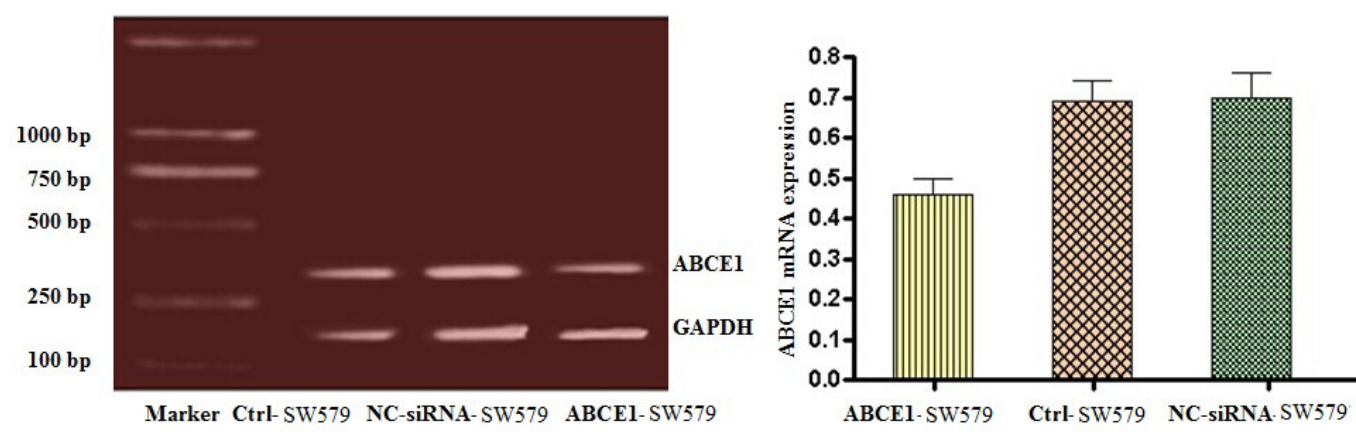

Figure 1. ABCE1-SW579 cells. ABCE1 mRNA expression decreased. Detection of ABCE1 mRNA expression in SW579 cells by reverse transcription polymerase chain reaction.

\section{siRNA inhibition of ABCE1 protein expression}

The results of the ABCE1 protein test are shown in Figure 2. The expression of $A B C E 1$ 
protein in the ABCE1-SW579 group was significantly reduced compared with the NC-SiRNA-SW579 and Ctrl-SW579 groups, and the differences were statistically significant $[(0.63 \pm 0.10)$ vs $(0.93 \pm$ $0.11)$, $(0.95 \pm 0.12) \mathrm{P}<0.05]$. There was no significant difference in ABCE1 protein expression between the Ctrl-SW579 group and the NC-siRNA-SW579 group ( $P>0.05$ ), which showed that $A B C E 1$-siRNA transfection effectively inhibits the expression of ABCE1 proteins in SW579 cells.
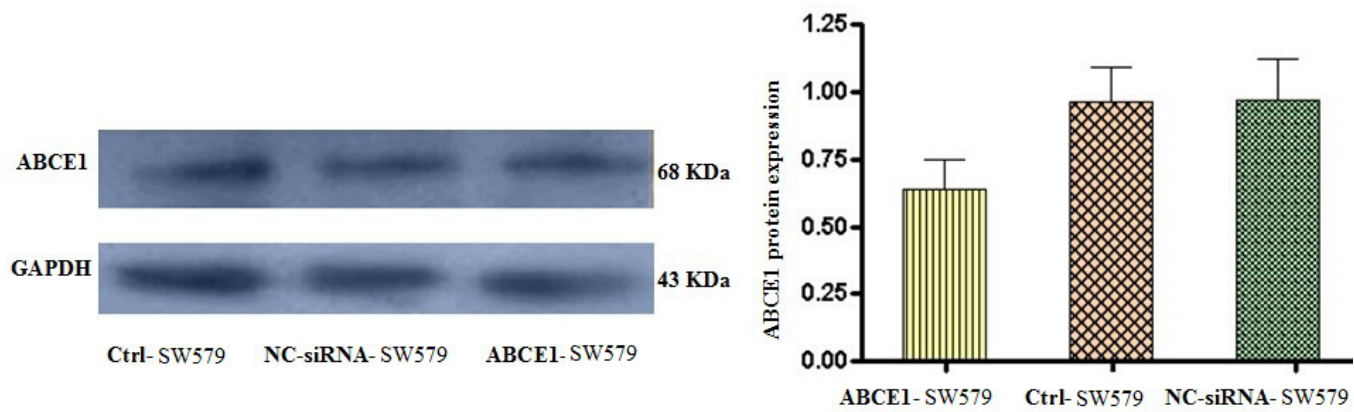

Figure 2. ABCE1 protein expression in the ABCE1-SW579 cells decreased.

\section{Cell cycle detection in ABCE1-SW579 cells using flow cytometry}

The effect of $A B C E 1$-siRNA on cell cycle was analyzed by flow cytometry, which showed that there were statistically significant differences in the number of cells at the G0/G1 and S-phases between the ABCE1-SW579 group and the Ctrl-SW579 group, and the ABCE1-SW579 group and the NC-siRNA-SW579 group $(P<0.05)$; there was no statistically significant difference between the Ctrl-SW579 group and the NC-siRNA-SW579 group in the number of cells at the G0/G1 phase or the S-phase $(\mathrm{P}>0.05)$, which shows that $A B C E 1$-siRNA arrests the cell cycle in the $\mathrm{G} 0 / \mathrm{G} 1$ phase (Table 1).

Table 1. Cell cycle distribution and apoptosis rate (\%) in each group.

\begin{tabular}{lcccc}
\hline Group & G0/G1 phase & S phase & G2/M phase & Apoptosis rate \\
\hline NC-siRNA-SW579 & $56.4 \pm 2.1$ & $35.3 \pm 2.6$ & $8.6 \pm 1.2$ & $0.61 \pm 0.23$ \\
Ctrl-SW579 & $54.5 \pm 2.0$ & $36.2 \pm 2.7$ & $9.1 \pm 1.1$ & $0.61 \pm 0.22$ \\
ABCE1-SW579 & $77.6 \pm 2.1^{*}$ & $16.2 \pm 1.1^{*}$ & $8.2 \pm 1.0$ & $17.89 \pm 0.84^{*}$ \\
\hline
\end{tabular}

${ }^{*} \mathrm{P}<0.05$, compared to Ctrl-SW578 or NC-siRNA-SW579 groups.

Table 2. Absorbance at $490 \mathrm{~nm}$ (means $\pm \mathrm{SD}, \mathrm{N}=6$ ).

\begin{tabular}{lcccc}
\hline Group & $48 \mathrm{~h}$ & $72 \mathrm{~h}$ & $96 \mathrm{~h}$ & $120 \mathrm{~h}$ \\
\hline NC-siRNA-SW579 & $0.6 \pm 0.03$ & $1.8 \pm 0.13$ & $2.5 \pm 0.17$ & $2.9 \pm 0.15$ \\
Ctrl-SW579 & $0.6 \pm 0.05$ & $1.7 \pm 0.12$ & $2.4 \pm 0.14$ & $2.8 \pm 0.16$ \\
ABCE1-SW579 & $0.6 \pm 0.06^{*}$ & $1.4 \pm 0.10$ & $1.8 \pm 0.12$ & $2.2 \pm 0.13$ \\
\hline
\end{tabular}

${ }^{*} \mathrm{P}<0.05$, compared to NC-siRNA-SW579 or Ctrl-SW579 group.

\section{ABCE1-SW579 cell apoptosis detected by flow cytometry}

Flow cytometry analysis showed that the cell apoptosis rate in the ABCE1-SW579 group 
was significantly higher than in the Ctrl-SW579 and NC-siRNA-SW579 groups, and the difference was statistically significant $(P<0.01)$. There was no significant difference in apoptosis rate between the Ctrl-SW579 and NC-siRNA-SW579 groups ( $>$ > 0.05) (Table 2).

\section{ABCE1-SW579 cell proliferation detected by the CCK-8 assay}

The growth curve of cells in the ABCE1-SW579 group, drawn according to the results of the CCK-8 assay, was significantly shallower than those of the Ctrl-SW579 and NC-siRNA-SW579 groups (Figure 3 ), with a statistically significant difference $(P<0.05)$.
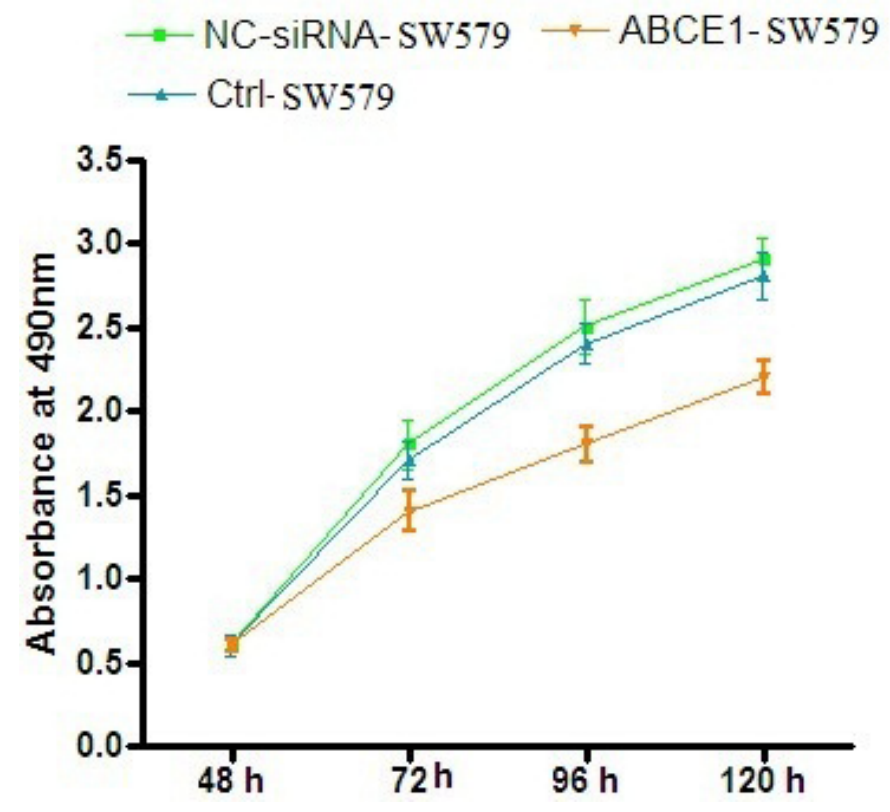

Figure 3. CCK-8 assay showed reduced ABCE1-SW579 cell proliferation. Growth curves of the three groups.

\section{Slow healing of ABCE1-SW579 cells detected by the cell scratch assay}

After 48 h, ABCE1-SW579 cells exhibited slow healing from scratches, while the scratches administered to the Ctrl-SW579 and NC-siRNA-SW579 groups had essentially healed, as shown in Figure 4.

\section{Reduction of invasiveness of ABCE1-SW579 cells in vitro}

The number of transmembrane cells in the Ctrl-SW579 and NC-siRNA-SW579 groups were $53.34 \pm 3.25$ and $54.27 \pm 4.21$, respectively (see Figure 5 ); the number in the ABCE1-SW579 group was significantly reduced $(31.80 \pm 3.78)$, and the difference was statistically significant $(P$ $<0.01)$. The results showed that specific interference in $A B C E 1$ gene expression may effectively reduce the invasiveness of SW579 cells. 

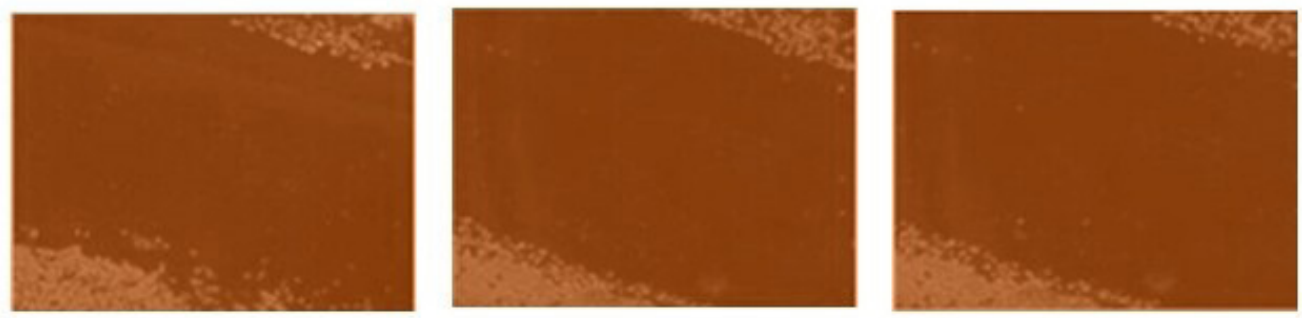

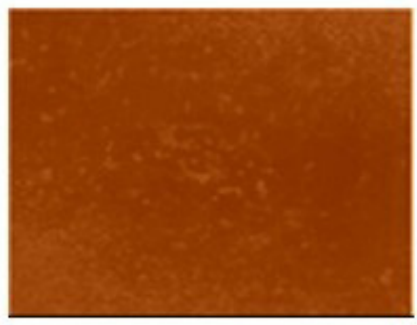

Ctrl-SW579

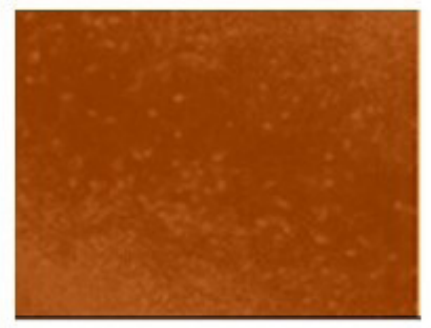

NC-siRNA-SW579

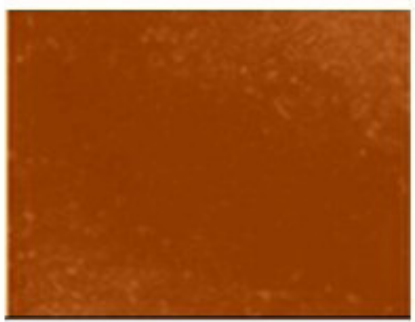

ABCEl-SW579

Figure 4. Cell scratch injury experiments. ABCE1-SW579 cells showed slow healing. As can be seen from Figure 4, after $48 \mathrm{~h}$, the ABCE1-SW579 cells showed slow healing of scratches, while scratches in the Ctrl-SW579 and NCsiRNA-SW579 groups had essentially healed, indicating that cell migration in the experimental group was significantly decreased (100X magnification).

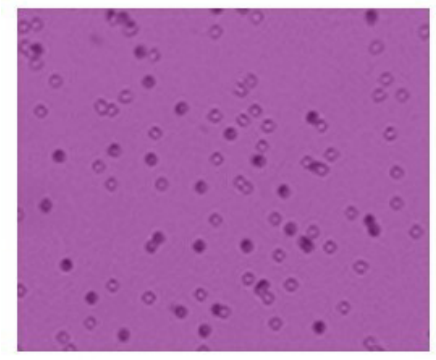

Ctrl-SW579

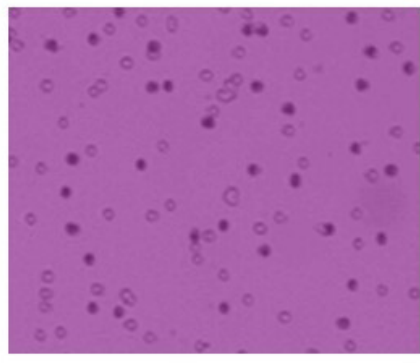

NC-siRNA-SW579

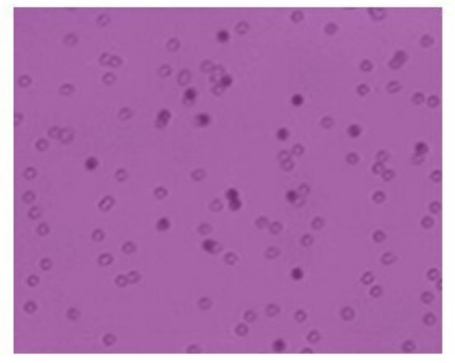

ABCE1-SW579

Figure 5. Reduction of ABCE1-SW579 cell invasion in vitro (200X magnification).

\section{DISCUSSION}

Thyroid cancer is a systemic disease; it is increasingly prevalent, and the age of onset is gradually decreasing. It can metastasize at an early stage via the lymphatic or cardiovascular systems, which poses a serious threat to women's health (Fokas et al., 2013). Both Chinese and international clinicians consider that surgery is the first choice for patients with thyroid cancer. Chemotherapy, radiotherapy, endocrine therapy, and comprehensive biomolecule-targeted therapy are supplemental treatments. However, local recurrence of thyroid cancer and metastasis affecting distant tissues and organs still pose huge clinical challenges. With the completion of the Human Genome Project and the development of functional genes, gene therapy applications in oncology 
are receiving more attention (Tian et al., 2012). Proliferation, invasion, and migration of thyroid cancer involve many abnormal gene structures and functions. Identifying the role of key genes in thyroid cancer is of great significance for the diagnosis, treatment, and prevention of thyroid cancer in a clinical context (Ren et al., 2012).

The ABCE1 protein is encoded by a member of the ATP-binding cassette multi-gene family, and the $A B C E 1$ gene has the locus $4 \mathrm{q} 31$ and a full-length cDNA sequence encoding 599 amino acids. ABCE1 has a mass of $68 \mathrm{kDa}$ and is expressed in every group of tissues and organs in the body (Ren et al., 2012). It inhibits the action of ribonuclease $L$, which normally binds to 2-5A (5'-phosphorylated 2',5'-linked oligoadenylates) and inhibits the 2-5A/RNase $L$ pathway. RNase $L$ causes the specific degradation of RNA, inhibits the $68-\mathrm{kDa}$ protein and the synthesis of normal proteins, induces apoptosis, and prevents the spread of viruses and the uncontrolled proliferation of cells (Bisbal et al., 2001). Inhibited proliferation of tumor cells is the key to apoptosis, invasion, and migration (Shimizu et al., 2013). Studies have shown (Terova et al., 2013) that the ABCE1 gene is an inhibitor of RNase L. RNase $L$ inhibits the interferon-regulated 2-5A/RNase $L$ pathway, which is used by viruses, influences the biological metabolism of normal cells, and induces apoptosis (Chen et al., 2012). Moreover, it can also promote cell proliferation, differentiation, and protein synthesis. Therefore, we speculate that it correlates closely with the biological behavior of tumor proliferation, invasion, and migration (van der Deen et al., 2005). Studies have shown that when the silencing ABCE1 gene is transfected into tumor cells, there is a significant change in their proliferation and apoptosis (Zheng et al., 2007). Other studies have shown that overexpression of the ABCE1 gene was found in lung cancer, colorectal cancer, and prostate cancer cells (Barthelme et al., 2007; Hühmer et al., 2013). However, literature on its effect on the biological behavior of thyroid cancer is scarce.

Electroporation is the preferred laboratory method for achieving high-efficiency transfection (Pisarev et al., 2010). Under the effect of high-voltage electric field pulses, tiny holes instantly appear in the cell membrane, and exogenous DNA can enter the cell. In this study, the designed and synthesized siRNA sequences ( $A B C E 1$-siRNA) of targeted $A B C E 1$ and the NC-siRNA were transfected into human thyroid cancer SW579 cells by electroporation, and the ABCE1-SW579 and NC-siRNA-SW579 cells were formed. Using RT-PCR and western blotting, we found that the expression levels of $A B C E 1$ mRNA and protein were blocked after transfection, confirming that the targeted $A B C E 1$ gene was silenced. Cell flow cytometry detected cell cycle and apoptosis in the cells. The CCK-8 proliferation assay, scratch healing assay, and cell invasion assay measured proliferation, migration, and the ability to attack human thyroid carcinoma SW579 cells. By observing the biological behavior of thyroid cancer SW579 cells, the results showed that the expression level of ABCE1 mRNA and protein in ABCE1-SW579 cells was significantly lower. The growth rate of ABCE1-SW579 cells was significantly reduced. The cell cycle was arrested in the $\mathrm{G} 0 / \mathrm{G} 1$ phase, and the numbers of cells in the $S$ phase decreased. The proliferation, invasion, and migration of ABCE1-SW579 cells decreased significantly, and the rate of apoptosis increased significantly.

The results show that in human thyroid carcinoma, $A B C E 1$ gene silencing can inhibit tumor cell proliferation, invasion, and migration, thereby providing an experimental basis for the genetic therapy of thyroid cancer.

\section{Conflicts of interest}

The authors declare no conflict of interest. 


\section{REFERENCES}

Barthelme D, Scheele U, Dinkelaker S, Janoschka A, et al. (2007). Structural organization of essential iron-sulfur clusters in the evolutionarily highly conserved ATP-binding cassette protein ABCE1. J. Biol. Chem. 282: 14598-14607.

Bisbal C, Salehzada T, Silhol M, Martinand C, et al. (2001). The 2-5A/RNase L pathway and inhibition by RNase L inhibitor (RLI). Meth. Mol. Biol. 160: 183-198.

Chen SH, Li D and Xu C (2012). Downregulation of Col1a1 induces differentiation in mouse spermatogonia. Asian J. Androl. 14: 842-849.

Fokas E, Weiss C and Rödel C (2013). The role of radiotherapy in the multimodal management of esophageal cancer. Dig. Dis. 31: 30-37.

Hühmer AF, Paulus A, Martin LB, Millis K, et al. (2013). The chromosome-centric human proteome project: a call to action. J. Proteome Res. 12: 28-32.

Pisareva VP, Skabkin MA, Hellen CU, Pestova TV, et al. (2011). Dissociation by Pelota, Hbs1 and ABCE1 of mammalian vacant $80 \mathrm{~S}$ ribosomes and stalled elongation complexes. EMBO J. 30: 1804-1817.

Pisarev AV, Skabkin MA, Pisareva VP, Skabkina OV, et al. (2010). The role of ABCE1 in eukaryotic posttermination ribosomal recycling. Mol. Cell. 37: 196-210.

Ren Y, Li Y and Tian D (2012). Role of the ABCE1 gene in human lung adenocarcinoma. Oncol. Rep. 27: 965-970.

Shimizu M, Zaninotto G, Nagata K, Graham DY, et al. (2013). Esophageal squamous cell carcinoma with special reference to its early stage. Best Pract. Res. Clin. Gastroenterol. 27: 171-186.

Terova G, Rimoldi S, Bernardini G and Saroglia M (2013). Inhibition of myostatin gene expression in skeletal muscle of fish by in vivo electrically mediated dsRNA and shRNAi delivery. Mol. Biotechnol. 54: 673-684.

Tian Y, Han X and Tian DL (2012). The biological regulation of ABCE1. IUBMB Life. 64: 795-800.

van der Deen M, de Vries EG, Timens W, Scheper RJ, et al. (2005). ATP-binding cassette (ABC) transporters in normal and pathological lung. Respir. Res. 6: 59.

Zheng MG, Tian DL and Huang B. (2007). Construction and identification of a plasmid expressing siRNAs in mammalian cells aimed at ABCE1 gene. J. China Med. Univ. 36: 697-699. 